\title{
Research on mixer testing technology based on vector characterized method
}

\author{
Bao Cui ${ }^{1, a}$, Shenxiang Gao ${ }^{2, b}$,Xiaoqing Shen ${ }^{3, c}$, Ying Huo ${ }^{4, d}$ \\ ${ }^{1}$ China Satellite Maritime Tracking and Control Department, Jiangyin, China \\ ${ }^{2}$ China Satellite Maritime Tracking and Control Department, Jiangyin, China \\ ${ }^{3}$ China Satellite Maritime Tracking and Control Department, Jiangyin, China \\ ${ }^{3}$ China Satellite Maritime Tracking and Control Department, Jiangyin, China \\ a $214431{ }^{b} 214431{ }^{c} 214431{ }^{d} 214431$
}

\begin{abstract}
Keywords: vector characterized; mixer; Vector Network Analyzer; phase; group delay.
Abstract. The necessity of vector characterized is given by comparison of common test methods for mixer. Based on the model proposed by Agilent company, establish a simplified model of the parameter $C$ and investigate the mixing product and jamming signal processing method. Through the analysis of phase for two-port model, pull the conclusion that the phase of test signal has nothing to do with local oscillator signal, that illustrates the feasibility of absolute phase measurement. Finally, the feasibility and validity of the model and analysis are demonstrated by constructing the measurement system.
\end{abstract}

\section{Introduction}

The mixer is a key component in RF or microwave communication system, can achieve the function of frequency conversion of communication line, and its characteristics influence the communication performance of the system directly. In the design, production and application of mixer, it is necessary to measure the performance parameters quickly and accurately, such as frequency conversion loss, phase, group delay.

Mixer is multi ports device which makes use of the characteristics of nonlinear, the input and output in different frequency, its receiving unit and a reference unit should to be locked in different frequency when measuring the parameters. Thus, the conventional methods could not meet the need of vector parameters' measurement. Furthermore, it is difficult to correct vector error of the test system because of different frequency of the input and output. Therefore, the measurement and error correction of vector parameters are always the challenges for mixer test.

\section{Comparison of common test method}

At present, the common mixer test method consists of scalar test method and vector test method ${ }^{[1-3]}$. Scalar method mainly includes testing method based on spectrum analyzer, power meter or scalar network analyzer; With Vector Network Analyzer(VNA) ${ }^{[4]}$ as the foundation, vector method is through a combination of different test device to complete measurement. Comparison of two categories method is shown in Table 1. 
Table 1 Comparison of common measure methods for mixer

\begin{tabular}{|c|c|c|c|c|}
\hline \multicolumn{2}{|c|}{ Method } & \multirow{2}{*}{$\begin{array}{c}\text { Composition of } \\
\text { measurement system } \\
\begin{array}{c}\text { Two signal generator }+\mathrm{a} \\
\text { spectrum analyzer and } \\
\text { isolator }\end{array}\end{array}$} & \multirow[t]{2}{*}{ Advantage } & \multirow{2}{*}{$\begin{array}{c}\text { Limitation } \\
\text { Manual test, the test process is } \\
\text { complex, can't measure the } \\
\text { transmission phase shift and } \\
\text { group delay, low accuracy and } \\
\text { can't be corrected }\end{array}$} \\
\hline \multirow{3}{*}{$\begin{array}{l}\text { Scalar } \\
\text { Method }\end{array}$} & $\begin{array}{l}\text { Frequency } \\
\text { Spectrograph } \\
\text { Method }\end{array}$ & & & \\
\hline & $\begin{array}{l}\text { Power Meter } \\
\text { Method }\end{array}$ & $\begin{array}{l}\text { Two signal generator }+\mathrm{a} \\
\text { power meter and isolator }\end{array}$ & - & $\begin{array}{l}\text { Can't measure the } \\
\text { transmission phase shift and } \\
\text { group delay, more uncertainty } \\
\text { and can't be corrected }\end{array}$ \\
\hline & $\begin{array}{l}\text { Scalar Mixer } \\
\text { Calibrate } \\
\text { (SMC) }\end{array}$ & $\begin{array}{l}\text { Vector network analyzer } \\
\text { for dual signal source, or } \\
\text { network analyzer+signal } \\
\text { generator }\end{array}$ & $\begin{array}{l}\text { The measurement device } \\
\text { and the process are } \\
\text { simple,can reduce the } \\
\text { mismatch effect by } \\
\text { calibration }\end{array}$ & $\begin{array}{l}\text { Can't measure the mixer } \\
\text { transmission phase shift and } \\
\text { group delay }\end{array}$ \\
\hline \multirow[b]{3}{*}{$\begin{array}{l}\text { Vector } \\
\text { Method }\end{array}$} & $\begin{array}{c}\text { Vector Mixer } \\
\text { Calibrate } \\
\text { (VMC) } \\
\end{array}$ & $\begin{array}{l}\text { VNA for dual signal } \\
\text { source+ }+ \text { parametric mixer } \\
\text { and calibration mixer }\end{array}$ & $\begin{array}{l}\text { Can measure absolute } \\
\text { group delay, high accuracy }\end{array}$ & $\begin{array}{c}\text { Test system and process are } \\
\text { complex, requires the match } \\
\text { mixers }\end{array}$ \\
\hline & SMC+Phase & $\begin{array}{c}\text { Four port and dual signal } \\
\text { source VNA+calibration } \\
\text { mixer }\end{array}$ & $\begin{array}{l}\text { Do not need reference } \\
\text { mixer, the accuracy is high }\end{array}$ & $\begin{array}{l}\text { Phase measurement only } \\
\text { supports fixed local mode }\end{array}$ \\
\hline & Series Method & $\begin{array}{l}\text { VNA+filter+signal } \\
\text { generator+two } \\
\text { mixers+power divider } \\
\quad \text { (Attenuator) }\end{array}$ & $\begin{array}{l}\text { Do not need reference } \\
\text { mixer, VNA can work in } \\
\text { the frequency } \\
\text { synchronization state for } \\
\text { testing }\end{array}$ & $\begin{array}{l}\text { The frequency conversion } \\
\text { response of "mixer- filter" } \\
\text { components must be } \\
\text { reversible and the parameters } \\
\text { are similar, the filter is needed } \\
\text { to remove the influence of } \\
\text { mixer clutter }\end{array}$ \\
\hline
\end{tabular}

As the table shows, although the scalar method can measure conversion loss and other parameters, it can not get the vector parameter, such as phase and group delay. Conversely, the vector method, based on VNA, not only can measure the vector parameter, but also obtain higher measurement accuracy. However, the measuring principle of VNA is S parameter network model, and the S parameter model is linear, which requires all signals frequency should be the same, otherwise the phase will lose its significance in the model. The difference of signal frequency and lots of nonlinear frequency products merged in output signal result in higher hardware requirements for VNA. The failure of the $\mathrm{S}$ parameter model makes the conventional vector calibration is not suitable for network analyzer, which increases the uncertainty for mixer test. To fix these problems, mature mixer test theory and method emerged quickly, for instance, the frequency deviation error model. Some relevant units have developed the dedicated mixer measurement program based on VNA. However, such VNA is expensive and difficult to widespread.

From the Tab. 1 we know that using calibration mixer can make the measurement get higher accuracy. Calibration mixer, Vector Characterized(VC), is used as the "through" calibration standard for phase calibration. Thus, the frequency deviation error model of transmission and tracking errors can be determined, which can eliminate the influence of reference mixer conversion loss and transmission phase. Therefore, how to obtain the vector parameter of mixer is the key to mixer measurement.

\section{Vector characterized method research}

The VC method, based on reflection measurement, is proposed earliest by Agilent company ${ }^{[5]}$. The basic idea of the method is automatic frequency conversion of reflection measurement, that the Intermediate Frequency(IF) signal, reflected back into mixer by reflective device, would mix frequency twice. Then, measuring the reflection coefficient of mixer RF input by VNA, and according to the 
one-port vector error model, calculating the mixer parameters, such as conversion loss of amplitude and phase response. The test system shows in Figure 1.

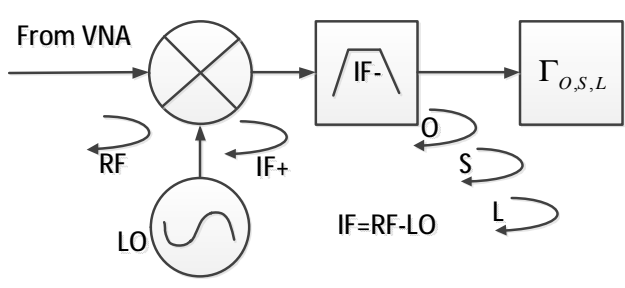

Fig. 1 Schematic diagram of the mixer vector characterized

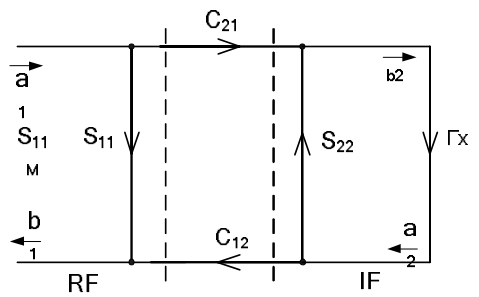

Fig. 2 Simplified C parameter model for mixer-filter component

The characterized object is a mixer-filter component, the test system just needs a VNA which can cover the RF range of mixer and a signal generator as the Local Oscillator(LO) .

Mathematical model of vector characterized. The mixer is a nonlinear device whose input and output are at different frequency, so the $S$ parameter model is not suitable for characterizing the mixer. The Agilent has proposed the $C$ parameter model of mixer ${ }^{[5]}$, but the model is too complicated. In order to simplify the analysis and calculation, this paper adopts a kind of flexible $S$ parameter model. The single port vector error model of the mixer-filter component is shown in Figure 2.

The left of dotted line is Radio Frequency(RF), and the right is Intermediate Frequency(IF). The variable frequency parameter is represented by $C$ in order to distinguish between the $S$ parameter model. The $\mathrm{S}_{11}$ and $\mathrm{S}_{22}$ represent input and output reflection coefficient, the module and angle of $\mathrm{C}_{21}$ respectively represent frequency conversion loss and transmission phase of the component. The $\Gamma_{x}$ represents the open circuit, short circuit and matched load connected with the intermediate frequency filter output port.

The voltage reflection coefficient by Mason formula

$$
S_{11 M}=\frac{b_{1}}{a_{1}}=S_{11}+C_{21} \frac{\Gamma_{x}}{1-S_{22} \Gamma_{x}} C_{12}
$$

Since the mixer response is reversible, so the expression $C_{21} \frac{\Gamma_{x}}{1-S_{22} \Gamma_{x}} C_{12}$ expresses the RF signal is changed back to the same frequency after twice frequency conversion. Accordingly, the (1) is a meaningful vector expression, which is the correct simplification of the $C$ parameter model.

The voltage reflection coefficient corresponding to the open circuit, short circuit and matched load are $\Gamma_{O}=1 、 \Gamma_{S}=-1$ and $\Gamma_{L}=0$, as the Eq. (1), let $C_{21}=C_{12}=T$, then

$$
\begin{aligned}
& S_{11}=S_{11 L} \\
& S_{11}=\frac{2 S_{11 L}-S_{110}-S_{11 S}}{S_{11 S}-S_{110}} \\
& T=\sqrt{\frac{2\left(S_{11 S}-S_{11 L}\right)\left(S_{110}-S_{11 L}\right)}{S_{11 S}-S_{110}}}
\end{aligned}
$$

The $S_{11 \mathrm{O}}, S_{11 \mathrm{~S}}$ and $S_{11 \mathrm{~L}}$ are corresponding to the voltage reflection coefficient of the open circuit, short circuit and matched load.

Study on the vector characterized of mixer

Processing of mixing product and jamming signal. The mathematical model (1) has an implicit condition that the driving signal of the second item (frequency conversion term) is a single frequency. But mixer is nonlinear device, and its output signal has many frequency components. Here, assuming the output frequency of mixer is

$$
f_{\mathrm{IF}}^{(1)}=m \cdot f_{\mathrm{RF}}^{(0)}+n \cdot f_{\mathrm{LO}}
$$


The $f_{\mathrm{RF}}^{(0)} 、 f_{\mathrm{IF}}^{(1)} 、 f_{\mathrm{LO}}$ respectively are the input signal of mixer input, the mixing product of mixer output and the LO signal, $m$ and $n$ are integers.

After reflection, the $f_{\mathrm{IF}}^{(1)}$ would input from the mixer output port and mix again, then the output signal would be:

$$
f_{\mathrm{RF}}^{(1)}=p\left(m \cdot f_{\mathrm{RF}}^{(0)}+n \cdot f_{\mathrm{LO}}\right)+q \cdot f_{\mathrm{LO}}=m p \cdot f_{\mathrm{RF}}^{(0)}+(n p+q) f_{\mathrm{LO}}
$$

In the (4), $f_{\mathrm{RF}}^{(1)}$ is the output signal in mixer input port which has been frequency conversion for two times, $p$ and $q$ are integers.

To make the $f_{\mathrm{RF}}^{(1)}=f_{\mathrm{RF}}^{(0)}$ set up, $m, p, q$ should meet the condition: $m=1, p=1, q=-n$, thus

$f_{\mathrm{IF}}^{(1)}=f_{\mathrm{RF}}^{(0)}+n \cdot f_{\mathrm{LO}}$

Namely, the mixing product of mixer, which meets the frequency in (5), would become $f_{\mathrm{RF}}^{(0)}$ after remixing. Except test signal, the others are jamming signals, and they would be filtered out by the filter. Thus, they will not become driving signals of the frequency conversion item in (1). The signal, reflected back by the filter, would be still become RF signal and appear at the input of mixer if meeting (5) and interfere measurement. These $f_{R F}$ are equivalent to the reflected signal of mixer input port in the mathematical model of this paper, result in increasing the value of $\mathrm{S}_{11}$.

Phase analysis of two port model of mixer filter module ${ }^{[6]}$. In order to get the absolute phase of mixer, the phase of every signal in network must be independent of the local oscillator. Assuming that the input RF signal and LO signal respectively are

$$
\begin{aligned}
& \mu_{R F}(t)=\cos \left(2 \pi f_{R F} t+\varphi_{R F}\right) \\
& \mu_{L O}(t)=\cos \left(2 \pi f_{L O} t+\varphi_{L O}\right)
\end{aligned}
$$

Based on the multiplier model of mixer, and just consider the frequency components of one or two order, the output signal of mixer would be

$$
\mu_{I F}(t)=C \mu_{R F}(t) \mu_{L O}(t)=\frac{|C|}{2} \cos \left(2 \pi\left(f_{R F}+f_{L O}\right) t+\varphi_{R F}+\varphi_{L O}+\phi\right)+\frac{|C|}{2} \cos \left(2 \pi\left(f_{R F}-f_{L O}\right) t+\varphi_{R F}-\varphi_{L O}+\phi\right)
$$

(7) $|C|, \phi$ are conversion loss and transmission phase shift of the reciprocal mixer.

There are five frequency components for the signal of network analyzer testing port: the mixer input port reflection signal $\mu_{R F 1}$, the sum frequency $\mu_{R F 2}$ and beat frequency $\mu_{R F 3}$ generated by $\mu_{I F+}$ and LO signal, the sum frequency $\mu_{R F 4}$ and beat frequency $\mu_{R F 5}$ generated by $\mu_{I F-}$ and LO signal, pass the analysis and calculation, it can obtain:

$$
\begin{aligned}
& \mu_{R F 1}=\left|\Gamma_{1}\right| \cos \left(2 \pi f_{R F} t+\varphi_{R F}+\phi_{1}\right) \\
& \mu_{R F 2}=\frac{|C|^{2}}{4} \cos \left[2 \pi\left(f_{R F}+2 f_{L O}\right) t+\varphi_{R F}+2 \varphi_{L O}+2 \phi\right] \\
& \mu_{R F 3}=\frac{|C|^{2}}{4} \cos \left(2 \pi f_{R F} t+\varphi_{R F}+2 \phi\right) \\
& \mu_{R F 4}=\frac{|C|^{2}\left|\Gamma_{2}\right|}{4} \cos \left(2 \pi f_{R F} t+\varphi_{R F}+2 \phi+\phi_{2}\right) \\
& \mu_{R F 5}=\frac{|C|^{2}\left|\Gamma_{2}\right|}{4} \cos \left[2 \pi\left(2 f_{L O}-f_{R F}\right) t+2 \varphi_{L O}-\varphi_{R F}+2 \phi+\phi_{2}\right]
\end{aligned}
$$

From the (8) we known that $\mu_{R F 1} 、 \mu_{R F 3} 、 \mu_{R F 4}$ have nothing to do with the phase of LO signal; The frequency of $\mu_{R F 2}$ is beyond the RF range of mixer; The frequency of $\mu_{R F 5}$ is three order, which is mirror frequency and can be filtered out by narrowing IF bandwidth. Thus, for mixer, whose mirror frequency response is filtered out, there are only $\mu_{R F 1} 、 \mu_{R F 3} 、 \mu_{R F 4}$ can be measured at the test port. 
Meanwhile, the frequency of them are $f_{R F}$ and all have nothing to do with the phase of LO signal. Therefore, the absolute phase is can be measured.

\section{Mixer test system based on vector characterized}

As shown in Figure 3, the test system is to make use of the reflectance measurement function of network analyzer, connect the calibrations (short circuit, open circuit and load) to the output port of mixer, then measure the reflection parameters (amplitude and phase) in mixer input port. According to the single port error model of VNA, the absolute group delay of mixer is calculated.

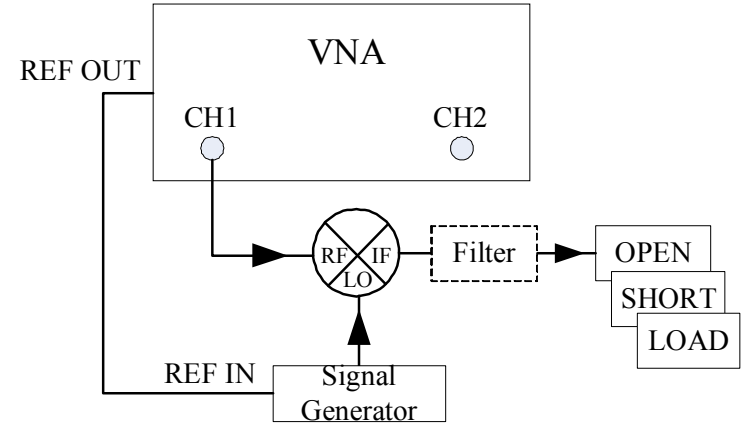

Fig. 3 Test system diagram of VC method

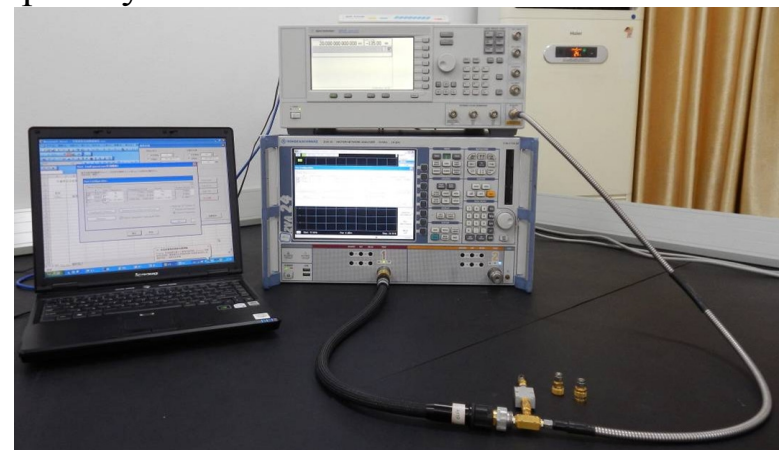

Fig. 4 Mixer test system based on VC

Hardware system. For the vector characterized mixer test, there just needs a basic configuration of VNA to complete the test, the test object is a mixer-filter component with reciprocity, the test system hardware is shown in Figure 4.

The frequency range of VNA and its calibration part should cover the range of mixer RF and IF frequency. The frequency range of the signal generator should cover mixer RF, and the maximum output level is greater than $10 \mathrm{dBm}$. In order to reduce influence of the measurement noise and the nonlinear frequency of mixer, the resolution bandwidth should be less than $1 \mathrm{kHz}$, and network analyzer and signal generator share the same reference frequency. The GP-IB bus is used by computer during measurement.

Testing software. In order to improve the efficiency and realize automatic test, the automatic test program is used to configure parameters of the instrument automatically, and the measurement results are collected and measured automatically as well. Based on the Excel spreadsheet, the background control code is compiled with VBA for the software program. The computing process is written in vector operation function, and the user interface shows in Figure 5.

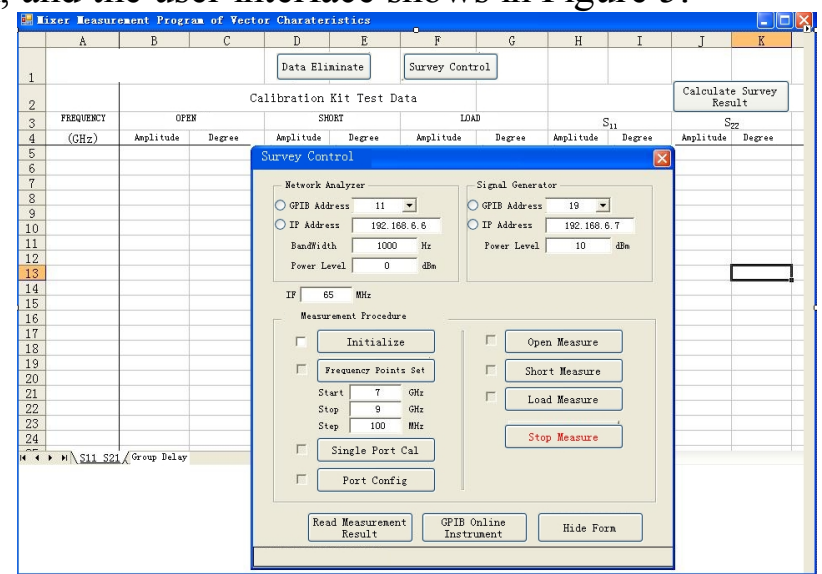

Fig. 5 Test software interface

\section{Result verification}

Verification process is to compare the results of $\mathrm{VC}$ with the results of PNA-X series network analyzer, calibrated by the SMC+Phase method. The phase calibration part of SMC+Phase calibration is comb generator. In order to eliminate the influence between different test instruments, two measurements use 
the same network analyzer. The test system shows in Fig. 4, and the configuration of device shows in Table 2.

Table 2 Test system configuration table

\begin{tabular}{|c|c|}
\hline Instrument name & Instrument model \\
\hline Vector network analyzer & N5242A \\
\hline Power meter & N1911A \\
\hline Power sensor & N1921A \\
\hline Phase reference standard & U9391C \\
\hline Electronic calibration parts & N4433A \\
\hline Mixer measured & TK2-18E2 \\
\hline Intermediate frequency filter & TKBPF-70-4S \\
\hline
\end{tabular}

Verification of the measurement results of reflection parameters and frequency conversion loss. The result curves of the two methods are shown in Figure 6, and the numerical comparison shows in Table 3. For ease of analysis, the data in Table 3 is the relative values for the comparison between two methods.

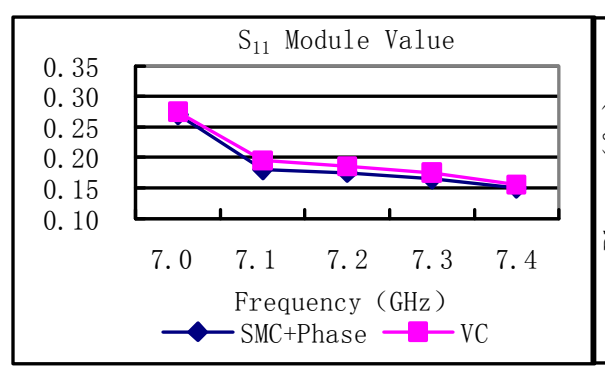

(a) $\mathrm{S}_{11}$ Module Value

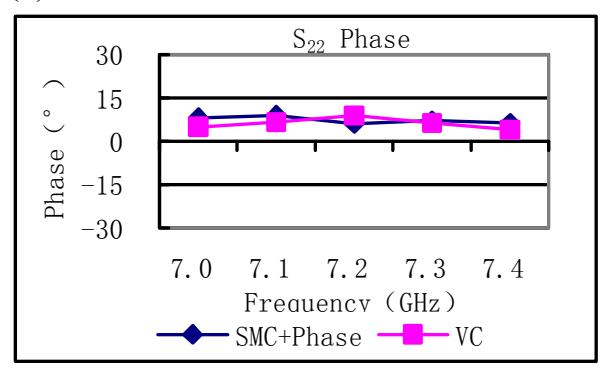

(d) $\mathrm{S}_{22}$ Phase

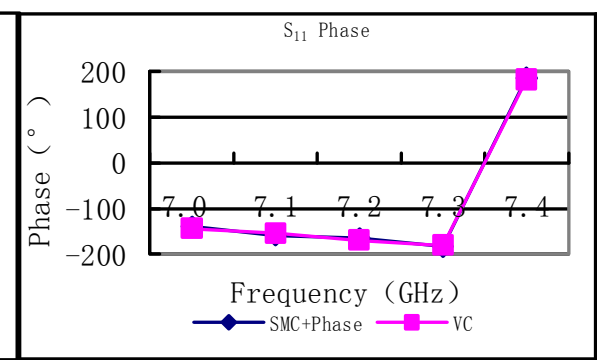

(b) $\mathrm{S}_{11}$ Phase

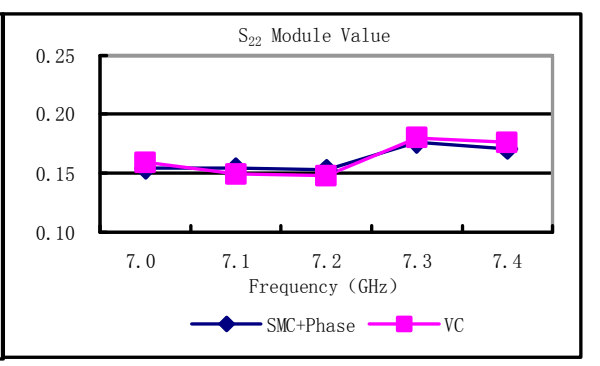

(c) $\mathrm{S}_{22}$ Module Value

Fig. 6 Comparison between the results of $\mathrm{VC}$ and $\mathrm{SMC}+\mathrm{Phase}$

Table 3 Relative error of VC for SMC+Phase

\begin{tabular}{|c|c|c|c|c|c|}
\hline $\begin{array}{c}\text { Frequency } \\
(\mathrm{GHz})\end{array}$ & $\begin{array}{c}S_{11} \text { Module } \\
\text { Value } \\
(\text { Relatively } \\
\text { ) }\end{array}$ & $\begin{array}{c}S_{11} \\
\text { Phase } \\
\left({ }^{\circ}\right)\end{array}$ & $\begin{array}{c}S_{22} \text { Module } \\
\text { Value } \\
(\text { Relatively } \\
\text { ) }\end{array}$ & $\begin{array}{c}S_{22} \\
\text { Phase } \\
\left({ }^{\circ}\right)\end{array}$ & $\begin{array}{c}C_{21} \text { Module } \\
\text { Value } \\
(\text { Relatively) }\end{array}$ \\
\hline 7.0 & $0.3 \%$ & -3.2 & $2.8 \%$ & -3.2 & $-1.9 \%$ \\
\hline 7.1 & $1.9 \%$ & -3.5 & $-1.6 \%$ & -3.5 & $-2.1 \%$ \\
\hline 7.2 & $1.2 \%$ & -2.5 & $-2.1 \%$ & 2.8 & $-1.8 \%$ \\
\hline 7.3 & $1.3 \%$ & -1.3 & $1.7 \%$ & -0.9 & $-2.6 \%$ \\
\hline 7.4 & $0.5 \%$ & -1.7 & $2.3 \%$ & -3.4 & $-2.4 \%$ \\
\hline
\end{tabular}

Pass the contrast, the $\mathrm{S}_{11}$ and $\mathrm{S}_{22}, \mathrm{C}_{21}$ module value error of $\mathrm{VC}$ method compared with the method of $\mathrm{SMC}+\mathrm{Phase}$ are less than $3 \%$, the phase measurement error is no more than 4 degrees, of which, for $\mathrm{S}_{11}$ module value, the measurement of VC method is slightly larger than that of SMC+Phase method, which is in agreement with the analysis of the 2.2.1 that $\mathrm{S}_{11}$ module value would increase slightly for simplified model. In conclusion, the mixer simplified $C$ model of VC method can be used to measure the reflectance parameters and conversion loss.

Verification of group delay measurement results. From Tab.1, we know that the mixer transfer phase shift could be measured precisely by series method. It needs to use connector when cascading two mixers, and the connector has large influence on phase measurement, but there is no influence in 
fixed frequency mode. Thus, the comparison of group delay between two methods would be in fixed frequency mode, and the comparison result is shown in Figure 7 and Table 4.

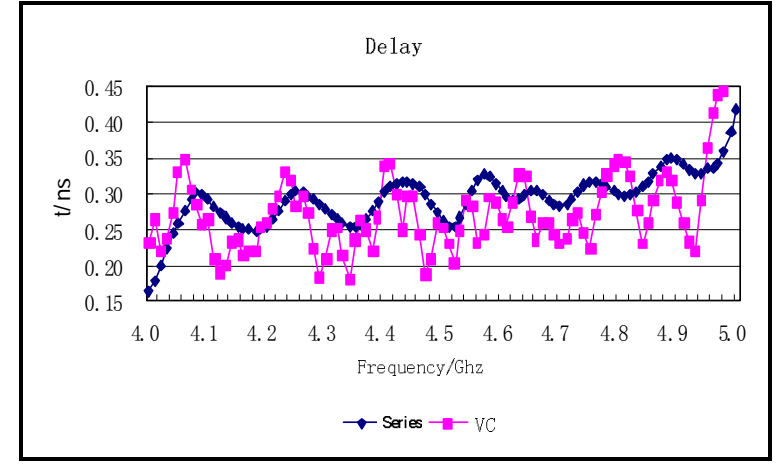

Table 4 Group delay measurement error of VC relative for series method

\begin{tabular}{|c|c|c|c|}
\hline \multirow{2}{*}{$\begin{array}{c}\text { Frequency } \\
(\mathrm{GHz})\end{array}$} & \multicolumn{3}{|c|}{ Delay (ns) } \\
\cline { 2 - 4 } & $\mathrm{VC}$ & Series & Error \\
\hline 4.0 & 0.22 & 0.18 & 0.04 \\
\hline 4.1 & 0.26 & 0.29 & -0.03 \\
\hline 4.2 & 0.26 & 0.26 & 0.00 \\
\hline 4.3 & 0.21 & 0.25 & -0.05 \\
\hline 4.4 & 0.34 & 0.30 & 0.04 \\
\hline 4.5 & 0.25 & 0.26 & -0.01 \\
\hline
\end{tabular}

Fig. 7 Group delay comparison of VC and series method

Pass the comparison, the maximum measurement error of the two methods is $0.05 \mathrm{~ns}$, means that the VC method can measure group delay accurately.

\section{Conclusion}

In recent years, the vector characterization of mixer is more and more applied in numerous advanced test method of the mixer. The vector characterized mixer, used as "pass through" calibration, can determine the frequency offset transmission error model of tracking error, can effectively eliminate the influence of reference mixer on the measurement of conversion loss and transmission phase shift. Test system only needs a covering RF range of vector network analyzer and a mixer as the local oscillator signal source. Test system can be built easily, and the measurement operation is simple. Therefore, it can be used for acceptance and debugging of the equipment, field test, etc.

\section{References}

[1] Jiwen Pan. A Research on Test Techniques for Microwave Mixer, Journal of Astronautic Metrology and Measurement(In Chinese). 2009, 29(5): 20-23.

[2] Zhiyong Sang, Shenxiang Gao, etc. Research on Frequency Conversion Loss Measurement Based on Mismatch Correction, Journal of Astronautic Metrology and Measurement(In Chinese). 2013, 33(4): 67-70.

[3] Xiang Song, Fushun Nian. Measurement of mixer based on vector network analyzer. Electronic Measurement Technology(In Chinese). 2011, 34(11): 113-117.

[4] Na Zhang. Application of vector network analyzer in particular measurement of the mixers. Foreign Electronic Measurement Technology(In Chinese). 2006, 25(10): 33-35.

[5] Dunsmore, Joel"Novel Method for Vector Mixer Characterization and Mixer Test System Vector Error Correction", Microwave Symposium Digest, 2002 IEEE MTT-S International, 2002, Page(s) 1833-1836 vol.3.

[6] Lu Han, Shenxiang Gao, Xiaoqing Shen. Research on Vector Characterization Measurement Method of Mixers. Journal of Astronautic Metrology and Measurement(In Chinese). 2012, 32(2): 50-53. 
